OPEN A Solution Processed Flexible

SUBJECT AREAS:

ORGANIC-INORGANIC NANOSTRUCTURES

ORGANIC LEDS

NANOPARTICLES

Received

9 January 2014

Accepted

17 February 2014

Published

17 March 2014

Correspondence and requests for materials should be addressed to

Q.B.P. (qpei@seas. ucla.edu)

* These authors contributed equally to this work.

\section{Nanocomposite Electrode with Efficient Light Extraction for Organic Light Emitting Diodes}

Lu Li*, Jiajie Liang*, Shu-Yu Chou, Xiaodan Zhu, Xiaofan Niu, ZhibinYu \& Qibing Pei

Department of Materials Sciences and Engineering, California NanoSystems Institute, Henry Samuli School of Engineering and Applied Science, University of California, Los Angeles, California 90095.

Highly efficient organic light emitting diodes (OLEDs) based on multiple layers of vapor evaporated small molecules, indium tin oxide transparent electrode, and glass substrate have been extensively investigated and are being commercialized. The light extraction from the exciton radiative decay is limited to less than $30 \%$ due to plasmonic quenching on the metallic cathode and the waveguide in the multi-layer sandwich structure. Here we report a flexible nanocomposite electrode comprising single-walled carbon nanotubes and silver nanowires stacked and embedded in the surface of a polymer substrate. Nanoparticles of barium strontium titanate are dispersed within the substrate to enhance light extraction efficiency. Green polymer OLED (PLEDs) fabricated on the nanocomposite electrode exhibit a maximum current efficiency of $118 \mathrm{~cd} / \mathrm{A}$ at $10,000 \mathrm{~cd} / \mathrm{m}^{2}$ with the calculated external quantum efficiency being $38.9 \%$. The efficiencies of white PLEDs are $46.7 \mathrm{~cd} / \mathrm{A}$ and $30.5 \%$, respectively. The devices can be bent to $3 \mathrm{~mm}$ radius repeatedly without significant loss of electroluminescent performance. The nanocomposite electrode could pave the way to high-efficiency flexible OLEDs with simplified device structure and low fabrication cost.

rganic light-emitting diodes (OLEDs) are currently being investigated for applications in large-areas flatpanel displays and solid state lighting ${ }^{1-3}$. Polymer OLEDs, or PLEDs offer potentially lower production cost and greater mechanical flexibility including elastomeric stretchability ${ }^{4-8}$. The introduction of a phosphorescent dopant in the organic emissive material allows for harvesting both singlet and triplet exciton energy and can increase the internal quantum efficiency $\left(\eta_{\mathrm{IQE}}\right)$ of the OLEDs to $100 \%^{3}$. However, the experimentally determined external quantum efficiency $\left(\eta_{\mathrm{EQE}}\right.$ ) has been limited to less than $30 \%$ without other out-coupling component ${ }^{9-11}$. The $70 \%$ of the produced photons is lost due to trapping inside OLEDs in the form of waveguide (WG) modes in organic/indium-tin oxide (ITO) transparent anode layers, surface plasmon-polariton (SPP) modes at the metallic electrode/organic interface, and glass substrate modes from total internal reflection at the glass substrate/ air interface ${ }^{12-15}$. The use of ITO and glass also affect the flexibility and fabrication cost of the OLEDs.

A number of techniques have been reported to increase the light extraction efficiency from modifying either the interface of organic/ITO layer and ITO/glass substrate (internal mechanism) or the glass substrate (external structure). The internal mechanisms use a meshed poly(3,4-ethylenedioxythiophene): poly(styrenesulfonate) (PEDOT:PSS) layer, low-index grids or nanoparticles ${ }^{16-18}$, micro-cavity ${ }^{19,20}$, surface plasmonic structures ${ }^{21,22}$ on the ITO layer, or embed photonic crystals, high-index optical coupling layer, dielectric mirrors, or buckled structures between ITO and glass to extract the waveguide mode in organic layer and ITO layer ${ }^{23-27}$. The internal mechanisms was to modify the light transport route and adjust the refractive index difference between the different functional layers. The methods could optimize the optical field and electrical field distribution in the organic layer and ITO layer to extract light. External structures are applied on the back surface of the substrate, including structured and shaped substrates ${ }^{28-32}$, scattering mediums $s^{33,34}$, and micro-lenses ${ }^{35,36}$. have also been shown to have a positive effect on the extraction of light out of the glass substrate. Also some strategy applied periodic or random nanowire electrodes ${ }^{37-39}$ to replace the ITO conductive electrode to prevent the ITO trapping mode. These approaches increase the light extraction efficiency to various extents, but the overall benefits have been limited due to an increased complexity in device fabrication, narrowed viewing angle, altered emission spectrum and/or angular dependency of the emission spectrum. Moreover, many of these approaches are not compatible with solution-based large scale industrial processing for flexible display and lighting panels. 
Here we report a solution processed flexible polymer nanocomposite conductor to replace ITO/glass for the fabrication of high-efficiency PLEDs. The nanocomposite comprises a layer of single-walled carbon nanotubes (SWNT) and a layer of silver nanowires (AgNWs) embedded in the surface of a barium strontium titanate (BST) nanoparticle-polymer nanocomposite. The SWNT layer on the outer layer of the nanocomposite interfaces with the polymer semiconductor layer(s) to smoothen the surface roughness and to increase the conductive surface coverage, while the AgNW layer underneath the SWNT provides long range electrical conductivity. The BST nanoparticles embedded in the polymer substrate disrupt the substrate mode of trapped light. Finite-difference time-domain
(FDTD) simulation indicates that the enhancement factor of the nanocomposite electrode as compared ITO/glass could be as high as $361 \%$. The experimentally obtained enhancement factors are $246 \%$ and $224 \%$ for green and white PLEDs, respectively, comparing the nanocomposite substrate to ITO/glass substrate. The maximum external quantum efficiency (EQE) of solution-processed green and white PLEDs are $36.7 \pm 2.2 \%$ and $28.8 \pm 1.7 \%$, respectively. Furthermore, these PLEDs are highly flexible and can be bent to $3 \mathrm{~mm}$ radius.

Figure 1 (a) illustrates the fabrication and structure of the flexible SWNT/AgNW nanocomposite. In a typical fabrication process, a glass substrate was used to provide a smooth release surface and

(a)
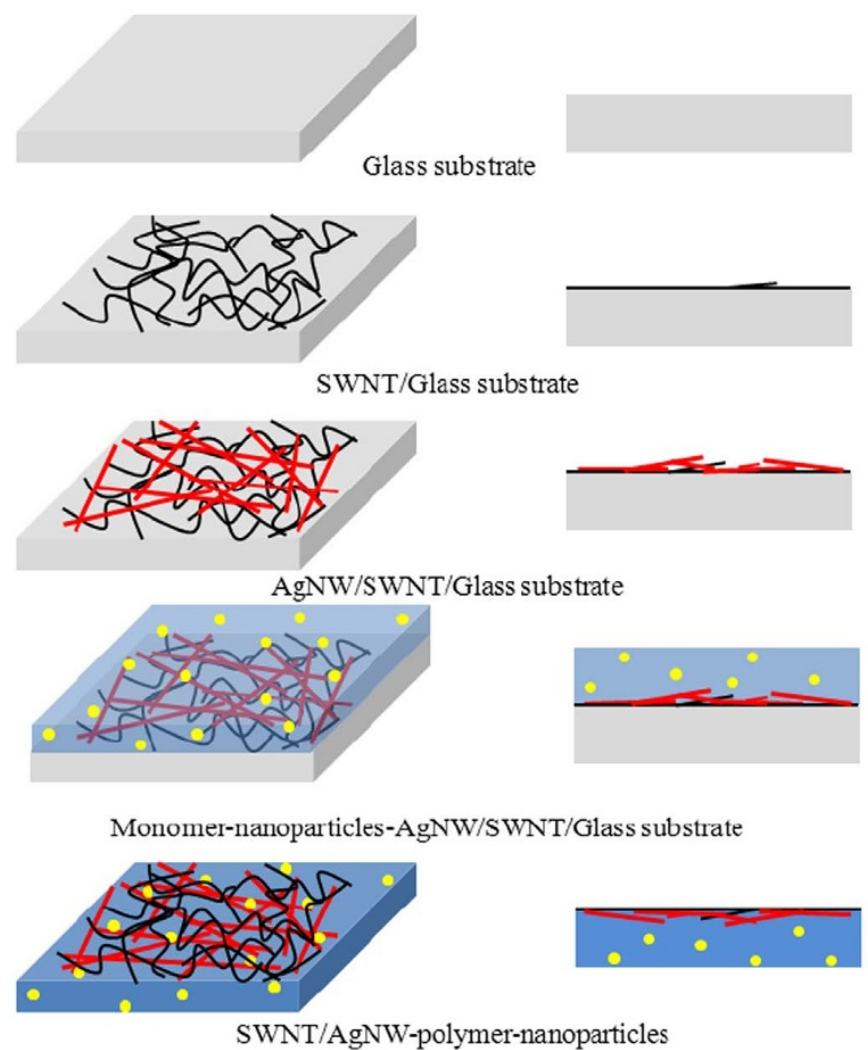

(b)

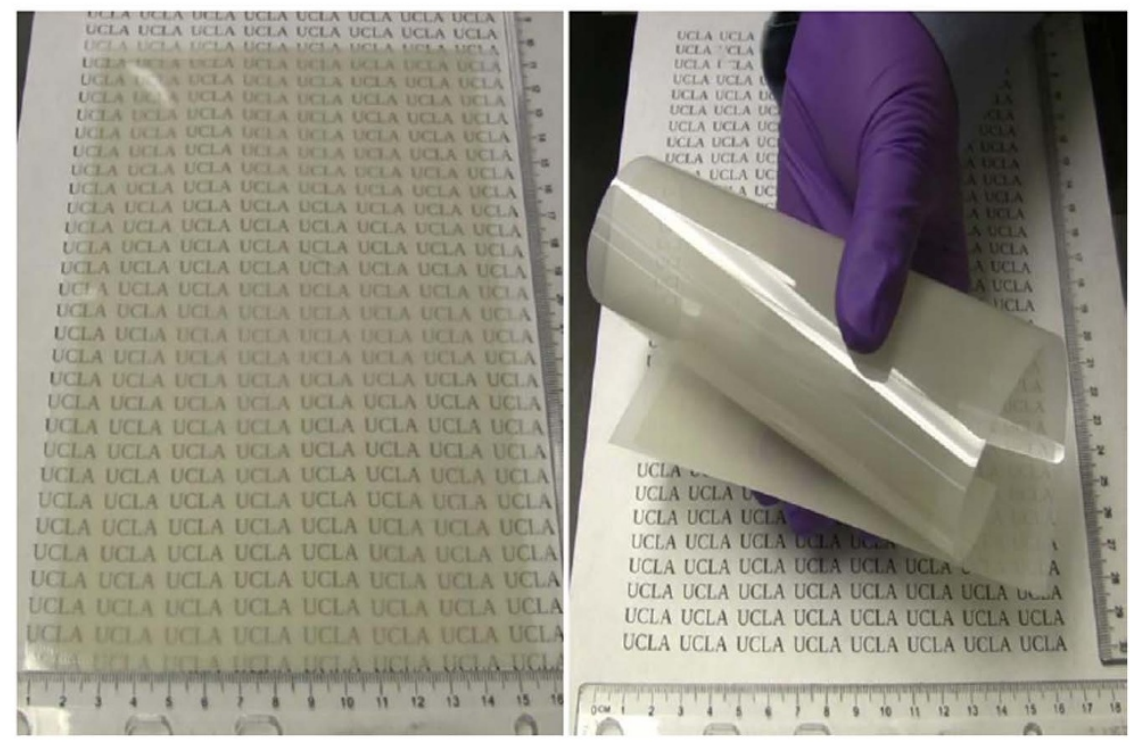

Figure 1 (a) Schematic illustration of the fabrication flowchart and structure of an SWNT/AgNW-nanocomposite electrode viewed from the top (left column) and cross-section (right column). Diagrams are not to scale; (b) Photographs of a nanocomposite film as prepared (205 $\mathrm{mm} \times 155 \mathrm{~mm}$ ). 
(a)

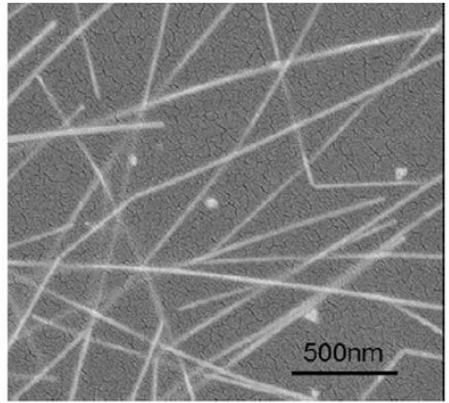

(c)

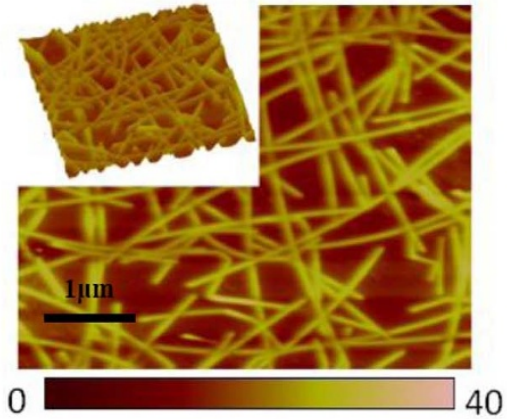

(e)

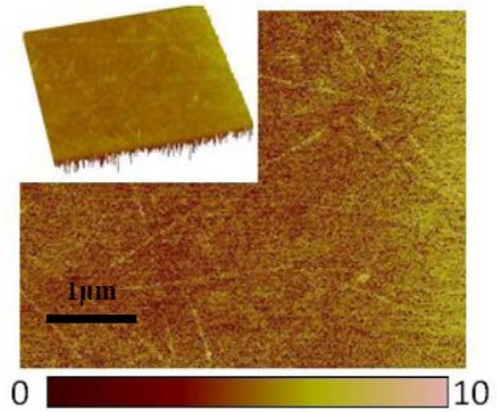

(b)

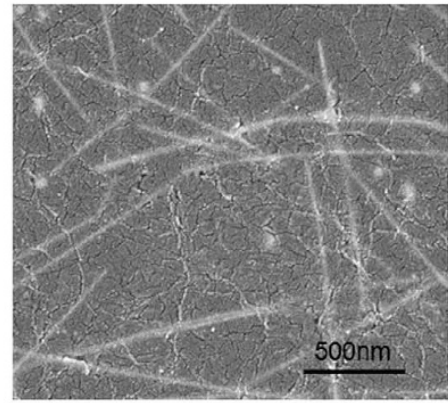

(d)

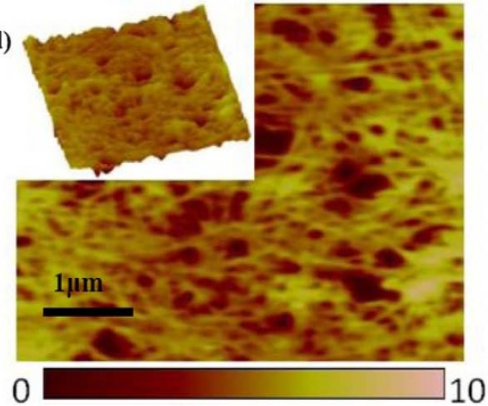

(f)
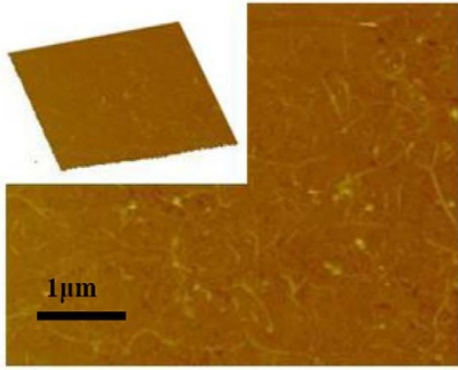

$0 \lcm{\square}$

Figure $2 \mid$ (a) SEM image of the conductive surface of an AgNW-nanocomposite. (b) SEM image of the conductive surface of an SWNT/AgNWnanocomposite film. Tapping mode AFM images and 3D topographical images (inset) of the conductive surface of AgNW-polymer (c), SWNT/AgNWpolymer (d), AgNW-nanocomposite (e), and SWNT/AgNW-nanocomposite (f).

dimensional stability. A dispersion of SWNTs in distilled water and isopropanol was coated onto the glass substrate, followed by coating of a dispersion of AgNWs in methanol onto the SWNT coating to form an AgNW/SWNT bilayer. The bilayer was thermally treated to form a highly conductive percolation network in the AgNW layer. A dispersion of BST nanoparticles in a diacrylate monomer solution was coated over the AgNW/SWNT bilayer which is highly porous. All the coatings were done using a Meyer rod. The nanoparticlemonomer coating was cured by ultraviolet radiation, forming a crosslinked nanocomposite film. The film was then peeled off the glass substrate. The AgNW/SWNT bilayer is transferred and embedded in the surface layer of the nanocomposite, with the SWNTs that were in contact with the release substrate exposed on the surface of the nanocomposite. The weight fractions of the AgNWs, SWNTs, and BST nanoparticles are all negligible compared to the diacrylate polymer which determines the mechanical properties of the resulting SWNT/AgNW-nanocomposite. Figure 1 (b) shows photographs of a nanocomposite film, $205 \mathrm{~mm} \times 105 \mathrm{~mm}$ area, laid flat on a piece of paper and rolled up to show its flexibility.

The distribution of SWNTs, AgNWs and BST nanoparticles in the nanocomposite was imaged using scanning electron microscopy (SEM). Fig. 2(a) shows the SEM image of the conductive surface of an AgNW-nanocomposite prepared by a similar method as the SWNT/AgNW-nanocomposite except that no SWNTs were employed. Both the nanowires and nanoparticles are sparsely and randomly distributed in the surface. In the
SWNT/AgNW-nanocomposite film (Fig. 2(b)), the SWNT layer appears uniform and densely distributed in the surface, in contrast to the sparse and random distribution of AgNWs and BST nanoparticles. The back surface of the nanocomposite (Fig. S1 (a)) does not contain any nanotubes or nanowires, but still shows the presence of a similar distribution of BST nanoparticles. The crosssectional image of the SWNT/AgNW-nanocomposite (Fig. S1 (b)) shows the distributions of the nanoparticles are sparsely distributed vertically in the bulk.

The surface morphology of the nanocomposites was further imaged using atomic force microscopy (AFM). Fig. 2 (c) to (f) shows the conductive surface of an AgNW-polymer, SWNT/AgNW-polymer, AgNW-nanocomposite, and SWNT/AgNW-nanocomposite films, respectively. In the acronyms, "polymer" indicates that no BST nanoparticles were employed in the poly(diacrylate) layer. The insets are the 3D images of the corresponding specimens. The AgNW-polymer film exhibits an AgNW network on the surface. "Hot-spots", or nanowires rising as much as $40 \mathrm{~nm}$ above the surface are observable. The average roughness $(\mathrm{Ra})$ is $7.62 \mathrm{~nm}$ which is higher than the $2.50 \mathrm{~nm}$ measured for the ITO/glass substrate used in this study. The surface roughness of the SWNT/AgNW-polymer electrode, prepared similarly to the SWNT/AgNW-nanocomposite except that no BST nanoparticles were employed, is much smaller, being $2.53 \mathrm{~nm}$, comparable to the surface of ITO/glass. As Fig. 2 (d) shows, the SWNT network completely covers the surface of the nanocomposite. The AgNW network is hardly visible in the AFM 
image. Another major difference between the AgNW-polymer and SWNT/AgNW-polymer electrodes is that AgNWs only cover a small fraction of the surface area in the former electrode. Electrical charges may not deliverable to the surface regions where there is no AgNW. The surface coverage in the SWNT/AgNW-polymer electrode is significantly improved for charge injection and collection throughout the entire surface area.

Admixing BST nanoparticles into the polymer matrix does not appear to increase the surface roughness. The Ra of AgNW-nanocomposite is $2.31 \mathrm{~nm}$, which is actually smaller than that of the AgNW-polymer. The $\mathrm{Ra}$ of the SWNT/AgNW-nanocomposite is only $1.51 \mathrm{~nm}$. The AFM image of the SWNT/AgNW-nanocomposite shows the SWNT network and some nanoparticles on the surface, whereas the AgNW network is completely buried and cannot be seen. This observation is consistent with bilayer the nanocomposite architecture wherein the SWNT network is exposed in the surface forming a dense conductive network with small surface roughness, in contrary to conventional coatings of SWNT on a substrate that forms a highly rough and porous surface. The AgNW percolation network is underneath the SWNT layer and improves the surface conductivity of the transparent anode. Note further that under ideal situation, the conductive surface of the nanocomposite should be a replica of the release substrate and could have extremely low roughness. In practice, the actual roughness depends on whether the monomers can diffuse and completely infiltrate all the nanopores within the coatings of the nanomaterials, and whether the bonding between the resulting polymer matrix and the nanomaterials is substantially stronger than the adhesion between the nanomaterials and the release substrate.

In order to develop an analytical approach that can quantitatively describe the effect of volume scattering on the out-coupling of an OLED, the assumption of radiative transport model and finite-difference time-domain (FDTD) method were employed. The scattering geometry for modeling was defined based on radiative transport model. We assumed that: i) the scattering layer (composite substrate) was infinite homogeneous and isotropic layer; 2) scattering events and the radiation source possess azimuthal symmetry; 3 ) the top surface is flat. This model has been developed extensively to describe scattering in different contexts and we take advantage of the considerable work on this topic $\mathrm{c}^{40,41}$. FDTD is a numerical analysis technique used for modeling computational electrodynamics and has been reported as a useful technique to simulate the optical behavior in the near field and far field regime through different types of substrates $^{42}$. Since it is a time-domain method, FDTD solutions can cover a wide frequency range with a single run and treat nonlinear material properties in a natural way. This method covers most of the important optical behaviors such as transmission, reflection, absorption, refraction and scattering. Using the FDTD method, the far field electric field intensity distribution of a dipole light source from the emissive layer in the present PLEDs to air was simulated based on ITO/glass and nanocomposite substrate, and the enhancement factor results are shown in Figure 3. Figure 3 (a) is a three dimensional color filled surface curve and its surface projection on the bottom. This figure displays the light extraction enhancement factor at $512 \mathrm{~nm}$ as a function of the size and refractive index of nanoparticles. The diameter of nanoparticle size is varied from $100 \mathrm{~nm}$ to $1000 \mathrm{~nm}$ and the refractive index from 2 to 3 . The light extraction enhancement factor reaches a maximum value of 3.61 when the nanopaticle diameter is $500 \mathrm{~nm}$ and refractive index is 2.6 (the doping concentration is fixed at $1 \mathrm{wt} \%$ ), which implies that a PLED based on nanocomposite substrate has the potential to be nearly 3.61 times as efficient as a conventional ITO-based device. Figure 3 (b) shows the light extraction enhancement factor of the nanoparticle with different sizes as a function of doping concentration from $0 \mathrm{wt} \%$ to $2.5 \mathrm{wt} \%$. The refractive index was set to 2.6 (the measured value for BST nanoparticles at $512 \mathrm{~nm}$ ). The peak enhancement factor for (a)

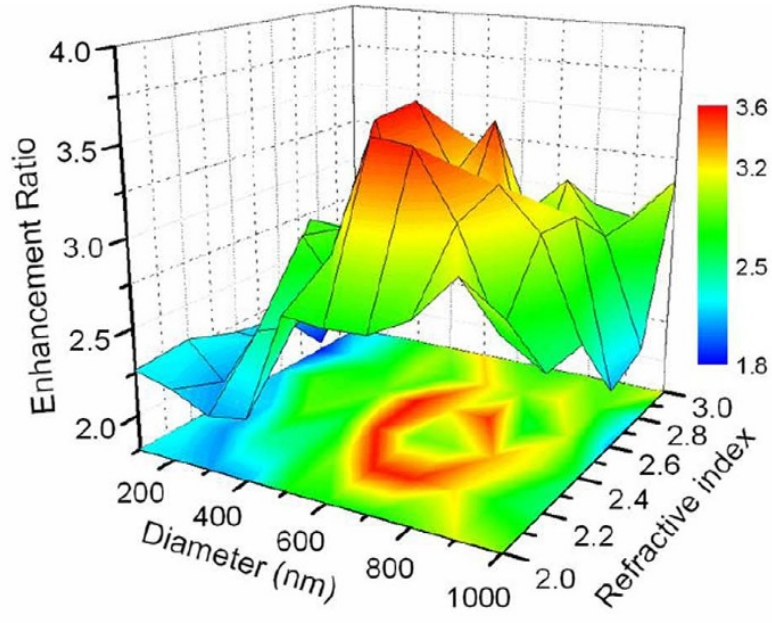

(b)

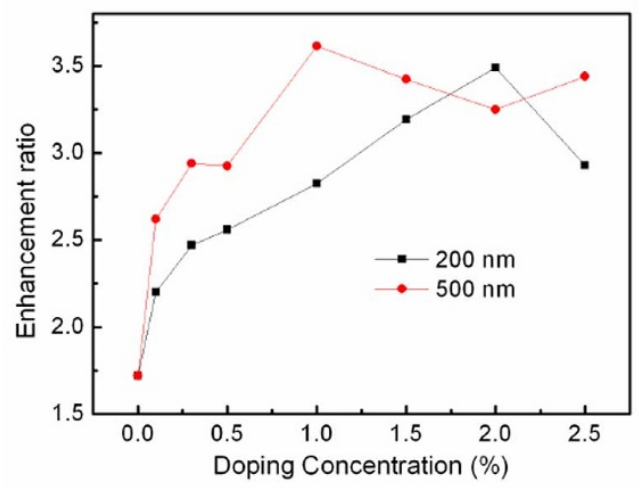

(c)

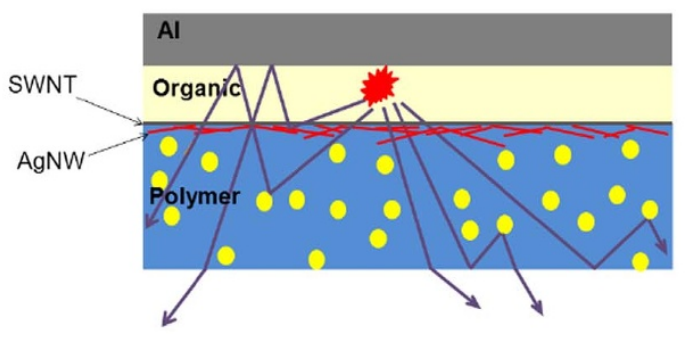

Figure $3 \mid$ (a) Simulated enhancement factor in light extraction efficiency as functions of the diameter and refractive index of nanoparticles dispersed in AgNW-nanocomposite substrate, as compared to ITO/glass in PLEDs. The doping concentration is fixed at $1 \mathrm{wt} \%$. (b) Simulated enhancement factors at various doping concentrations. The sizes of the nanoparticles are $200 \mathrm{~nm}$ or $500 \mathrm{~nm}$. The refractive index is 2.6. (c) Schematic illustration of light scattering by nanoparticles in the SWNT/AgNW-nanocomposite.

the $200 \mathrm{~nm}$ diameter nanoparticles is $349 \%$ reached at $2 \mathrm{wt} \%$ concentration.

Fig. 3 (c) is a schematic representation of the light scattering modes in a PLED based on the nanocomposite substrate. It overcomes two of the major light trapping modes in PLEDs with ITO/ glass as the transparent anode: (1) the waveguide mode in the ITO layer is eliminated; and (2) glass substrate mode is suppressed as the light propagation direction can be altered by the nanoparticles and nanowires. The absorption and surface plasma mode on the metal cathode is still present in the nanocomposite-based PLED. For PLEDs with AgNW-polymer substrate, the enhancement of light extraction has been attributed to high transmittance of the composite substrate, high build-in potential and light scattering from the AgNW surface ${ }^{38,39,43}$. Better contact with the organic emissive layer and higher surface coverage have also been suggested for SWNT/ AgNW-polymer substrate to enhance PLED performance ${ }^{44}$. For the 
(a)

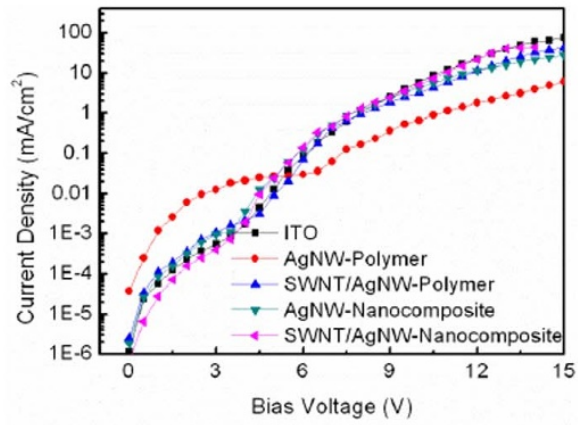

(c)

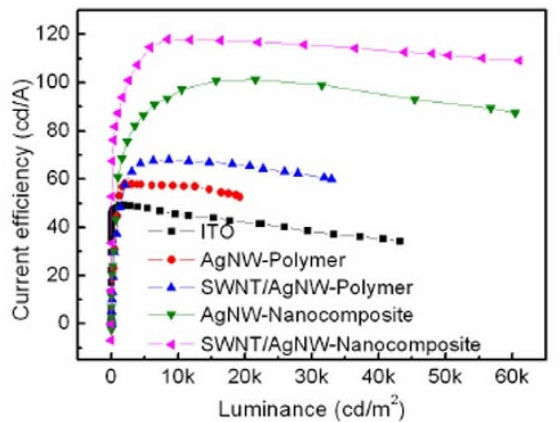

(e)

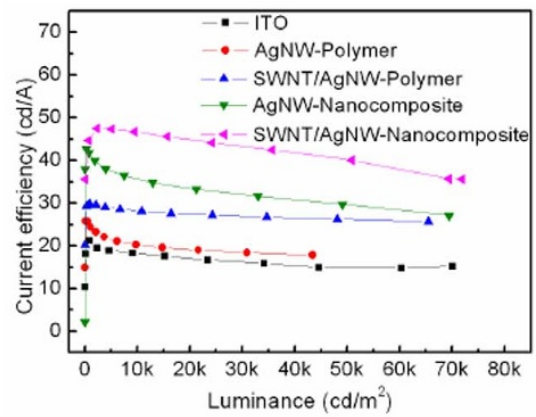

(g)

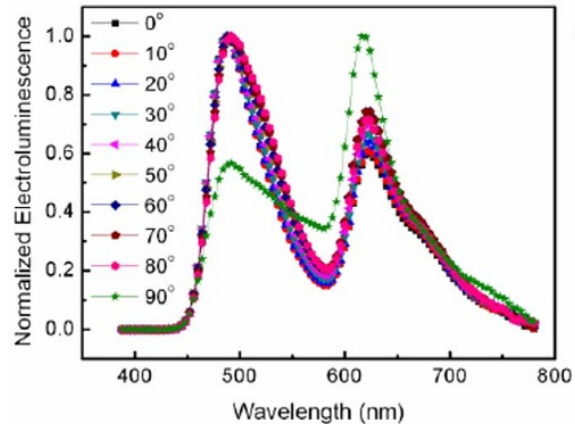

(i)

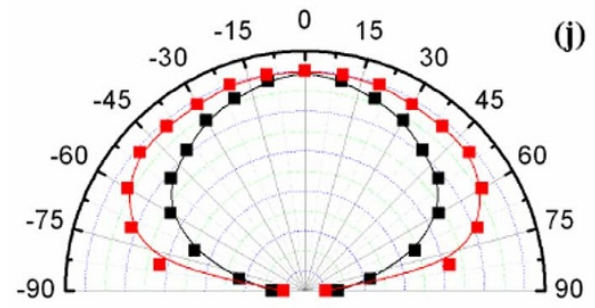

(b)

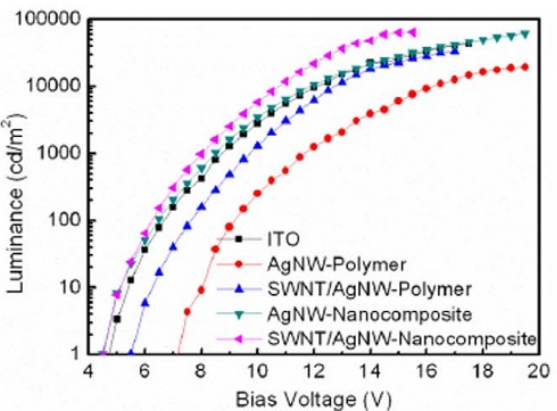

(d)

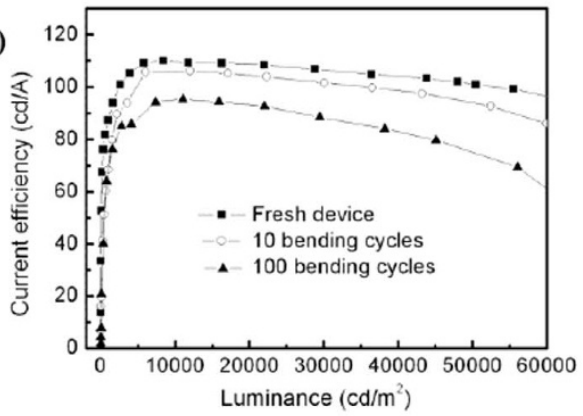

(f)

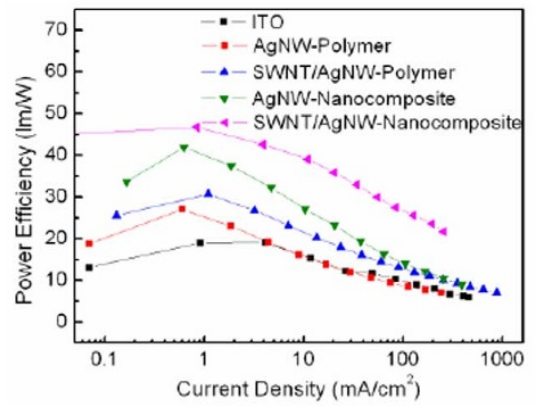

(h)

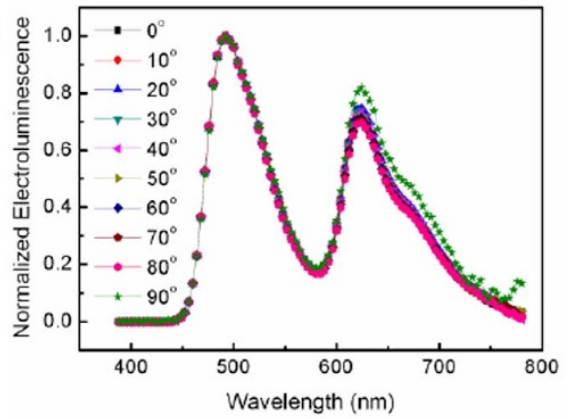

(j)
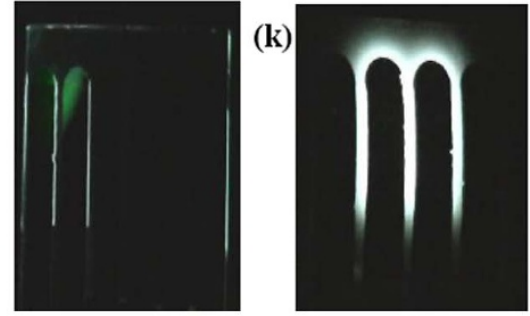

Figure 4 | Current density-bias voltage (a), luminance-bias voltage (b) and current efficiency-luminance (c) characteristics of green PLEDs based on specified substrates; Current density-luminance characteristics of a SWNT/AgNW-nanocomposite based PLED before, after 10 and 100 cycles of bending to $3 \mathrm{~mm}$ radius (d); current efficiency-luminance characteristics of white PLEDs (e) based on specified substrates; Power efficiency-current density characteristics (f) the white PLEDs; EL spectra measured at specified viewing angles of PLEDs based on ITO/glass substrate (g) and SWNT/AgNWnanocomposite substrate (h); (i) Angular distribution of the luminance of the devices based on ITO/glass substrate (black dot) and SWNT/AgNWnanocomposite substrate (red square); Photographs of white devices based on ITO/glass substrate ( $\mathrm{j}$ ) and composite substrate (k), taken from the cathode side working at $5000 \mathrm{~cd} / \mathrm{m}^{2}$. 


\begin{tabular}{|c|c|c|c|c|c|}
\hline & Structure & Efficiency $(c d / A)^{[a]}$ & Efficiency $(c d / A)^{[b]}$ & $\begin{array}{l}\text { Power efficiency }(\mathrm{lm} / \\
\text { W) }\end{array}$ & EQE (Best EQE) $(\%)^{[d]}$ \\
\hline
\end{tabular}

SWNT/AgNW-nanocomposite substrate, while these enhancement factors are preserved, the addition of dielectric nanoparticles provides an independently tunable scattering factor to maximize the light extraction efficiency of PLEDs ${ }^{27,33,42,45,46}$.

To demonstrate the enhancement in light extraction, green PLEDs with a sandwich structure of Al/CsF/Emissive layer/PEDOT:PSS $(40 \mathrm{~nm})$ /anode-substrate were fabricated. The emissive layer comprised poly(vinylcarbazole) (PVK), 1,3-bis[(4-tert-butylphenyl)-1,3, 4-oxidiazolyl]phenylene (OXD-7), and tris(2-(4-tolyl)pyridinato$\mathrm{N}, \mathrm{C} 2)\left(\operatorname{Ir}(\mathrm{mppy})_{3}\right)$ admixed at $100: 40: 0.5$ weight ratio. PVK is selected as the phosphorescence host because of its high triplet energy level $(3.0 \mathrm{eV})$, good film-forming property, high glass transition temperature $\left(\mathrm{T}_{\mathrm{g}} \sim 160^{\circ} \mathrm{C}\right)$ and the ability to conduct holes. White PLEDs were also fabricated with a similar device structure except that the emissive layer is a blend of a White Polymer (WP) and OXD-7 at 100:40 weight ratio. Cesium fluoride (CsF) and aluminum act as the electron injection material and cathode, respectively. The PLED structure and composition were initially optimized to obtain the highest efficiency for control PLEDs on ITO/glass. Five different anode-substrate compositions were then investigated: (1) ITO/glass (thickness: $150 \mathrm{~nm} / 0.6 \mathrm{~mm}$; sheet resistance: $15 \mathrm{ohm} / \mathrm{sq}$ ), (2) AgNW-polymer (30 ohm/sq), (3) SWNT/AgNW-polymer (50 $\mathrm{ohm} / \mathrm{sq}$ ), (4) AgNW-nanocomposite (200 nm nanoparticles, $2 \mathrm{wt} \%$ concentration, $50 \mathrm{ohm} / \mathrm{sq}$ ), and (5) SWNT/AgNW-nanocomposite (diameter $200 \mathrm{~nm}$ nanoparticles, $2 \mathrm{wt} \%, 50 \mathrm{ohm} / \mathrm{sq}$ ). BST nanoparticles with $200 \mathrm{~nm}$ diameter were used in this experiment due to ease of dispersion in the diacrylate monomer.

Figure 4 (a) shows the current density-bias voltage and luminance-bias voltage characteristics of the green PLEDs. The current densities at given voltages are almost identical for most PLEDs except for the device based on the AgNW-polymer substrate. The relative current densities at voltages below light emission threshold follow the same trend as the surface roughnesses for different types of substrate. The device based on AgNW-polymer has the highest current density as this substrate has the largest surface roughness as indicated by AFM imaging, and hence highest leakage current. The SWNT/AgNW-nanocomposite based PLED has the lowest leakage current. Above the threshold, the AgNW-polymer based device has the lowest current density, whereas the SWNT/AgNW-nanocomposite and ITO/glass based PLEDs have among the highest current density.

Figure 4 (b) shows that the luminance is increased and turn-on voltage reduced by adding SWNT and BST nanoparticles in the polymeric substrate. The turn-on voltage of the device based on AgNW-polymer is much higher than the other devices. This high turn-on voltage and the low current density described above are attributed to the low surface coverage of AgNWs and high surface roughness. The exponential increase of current density in PLEDs above turn-on is resulted from the dual injections of electrons and holes ${ }^{47}$. Below the electroluminescence turn-on, current injection, excluding leakage, is usually dominated by either holes or electrons which induces large space-charge accumulation. The low surface coverage of AgNWs in the AgNW-polymer should have a negative effect on delivering holes onto the areas uncovered by AgNW and lower the current density of hole injection into the emissive layer. The PEDOT:PSS layer has a rather low conductivity to deliver holes at high density over 100's nm range. When a layer of SWNT is applied onto AgNW, the surface coverage is greatly improved. Therefore the turn-on voltage and luminance are both increased in the SWNT/AgNW-polymer based PLED. The addition of BST nanoparticles further lowers the turn-on voltage and increases the luminance, more so than even the ITO/glass based device, thanks to enhanced light extraction efficiency. Figure 4 (c) shows the current efficiency-luminance characteristics of the green PLEDs. The characteristics of the device performance are summarized in Table 1. For the green devices (1) through (5), the current efficiency at 10,000 cd/ $\mathrm{m}^{2}$ is $46.8 \pm 2.2,55.7 \pm 2.3,66.0 \pm 2.7,94.8 \pm 6.5$, and $111.1 \pm$ $7.1 \mathrm{~cd} / \mathrm{A}$, respectively. All of the flexible green PLEDs based on the polymer and nanocomposite electrodes show various degrees of improvement in current efficiency compared to those on ITO/glass substrate. The percentages of improvement over (1) are $18.3 \%$, $40.0 \%, 106 \%$, and $146 \%$ for (2) through (5), respectively. This trend is consistent with the prediction of the FDTD simulation. The highest EQE is $38.9 \%$ for the PLEDs based on the SWNT/AgNWnanocomposite.

The enhancement factors of the nanocomposite electrode are generally lower than the simulation results. The discrepancy could be caused by the difference in the refractive indices of the emissive polymer layer and the nanocomposite, the difference in the shape and locations of the nanoparticles and nanowires. The enhancement factor of nanocomposite substrate with different nanoparticles loading can be seen in Figure S2. With the doping concentration increased from $0.1 \mathrm{wt} \%$ to $2 \mathrm{wt} \%$, the difference of simulated and measured enhancement factor is increased.

In the test of mechanical flexibility, PLEDs based on flexible nanocomposite substrate were bent to concave and convex curvatures with $3 \mathrm{~mm}$ radius. Figure 4 (d) shows the current efficiency characteristics of an SWNT/AgNW-nanocomposite based PLED before, after 10 and 100 cycles of bending to $3 \mathrm{~mm}$ radius. After 10 cycles of bending, the electroluminescent responses show only a little degradation. The current efficiency was reduced by only $5 \%$ compared to the fresh device. After 100 cycles, noticeable degradation is observed. The maximum current efficiency has reduced from $110 \mathrm{~cd} / \mathrm{A}$ to $92 \mathrm{~cd} / \mathrm{A}$. The degradation mechanism is the subject of 
further investigation. We suspect that delamination between the active layer and cathode may have occurred during repeatedly bending ${ }^{43,48}$.

For the white PLEDs, the current density-bias voltage-luminance characteristics are shown in Figure S3, and similar degrees of enhancement in current efficiency and $\mathrm{EQE}$ are obtained as the green PLEDs. Figure 4 (e) and (f) show the current efficiency and power efficiency of the white PLEDs, respectively. The maximum EQE of $28.8 \pm 1.7 \%$ is lower than the corresponding green PLEDs, as the white emission is a combination of fluorescence (blue) and phosphorescence (red). The emission spectra of the white PLEDs on ITO/ glass and SWNT/AgNW-nanocomposite substrate are nearly identical as shown in Figure S3.

The power efficiency of the white PLEDs (1) through (5) as a function of current density is displayed in Figure 4 (f). The maximum efficiencies are $18.3 \pm 0.7 \mathrm{~lm} / \mathrm{W}, 26 \pm 1.0 \mathrm{~lm} / \mathrm{W}, 29.7 \pm 1.0 \mathrm{~lm} / \mathrm{W}$, $40.1 \pm 1.8 \mathrm{~lm} / \mathrm{W}$, and $44.0 \pm 2.8 \mathrm{~lm} / \mathrm{W}$, respectively. The enhancement factor as a result of replacing ITO/glass with the SWNT/ $\mathrm{AgNW}$-nanocomposite is $246 \%$. Characteristic values are also shown in Table 1 based on the results of 10 devices for each substrate.

The spectra of the green and white PLED based on the SWNT/ AgNW-nanocomposite are identical to those based on ITO/glass (Figure S4). Figure 4 (g) and (h) show the normalized EL spectra at different viewing angles for the white PLEDs based on ITO/glass substrate and nanocomposite substrate. The normalized spectra show changes of the ratio of red to blue and the color quality of the light emission at different viewing angles. For the device on ITO/glass, the ratio of red to blue increases with increasing viewing angles from $0^{\circ}$ to $80^{\circ}$, and the white light becomes more warm white light. The side emission (at $90^{\circ}$ angle) is totally different. The corresponding change in the CIE cooordinates is shown in Figure S5. For the white PLEDs based on nanocomposite substrate, there is almost no change in the emission spectrum all viewing angles including $90^{\circ}$. The same phenomena are shown in Figure S6 for green PLEDs. This high color stability invariant with viewing angle is especially useful for lighting applications in the corner and on the roof.

Figure 4 (i) shows the normalized brightness as a function of viewing angle for the white PLEDs. The angular dependence of the device based on the nanocomposite electrode is quite different from the device on ITO/glass. The latter is characteristic of a Lambertian emission. For the SWNT/AgNW-polymer-BST nanocomposite sample, at $80^{\circ}$ and $-80^{\circ}$ viewing angles the luminance is $62 \%$ of that at $0^{\circ}$ (normal to device surface). Figure $4(\mathrm{j})$ and $(\mathrm{k})$ are photographs of the devices taken from the backside of the Al cathode operating at $5000 \mathrm{~cd} / \mathrm{m}^{2}$. The ITO/glass PLED has little emission visible from the backside, a result of weak emission at large angles at the front side. The nanocomposite-based PLED shows bright wide-angle emission. Similar observations were obtained with the green PLEDs (see Figure S7).

In summary, a flexible nanocomposite electrode has been prepared with high light extraction efficiency and useful for the fabrication of high-efficiency, solution-processed PLEDs. The nanocomposite comprises a bilayer stack of SWNT and AgNWs inlaid in the surface of a nanoparticle-polymer composite membrane, and has high surface conductivity and low surface roughness. FDTD simulation shows that the light extraction efficiency of the resulting PLEDs can potentially reach $361 \%$ over control devices on ITO/glass. The experimentally obtained enhancement factor is $246 \%$ for green PLEDs and 224\% for white PLEDs. The maximum current efficiency of green PLEDs is $111.1 \pm 7.1 \mathrm{~cd} / \mathrm{A}$, corresponding to an $\mathrm{EQE}$ of 36.7 $\pm 2.2 \%$ which is significantly higher than the reported highest $\mathrm{EQE}$ for all OLEDs without external light collection structure, including small molecule OLEDs comprising multiple layers of vacuum-deposited organic compounds to increase the internal quantum efficiency to unity. The maximum EQE of white PLEDs is $28.8 \pm 1.7 \%$ and the maximum luminous efficacy is $44 \pm 2.8 \mathrm{~lm} / \mathrm{W}$. The viewing angle of the PLEDs is also improved such that the emission color from the $90^{\circ}$ angle is almost identical to the normal angle. The nanocomposite electrode is promising to replace both the internal and external light extraction mechanisms introduced to enhance the performance of OLEDs based on ITO/glass.

\section{Methods}

AgNW/SWNT bilayer films were deposited by successively coating a layer of functionalized SWNT (Carbon Solutions, Inc.) and a layer of AgNW (synthesized according to a literature procedure ${ }^{49-51}$.) from respective dispersions at ambient temperature and pressure. Ethoxylated bisphenol-A dimethacrylate (DMA, Sartomer USA) and 2,2-dimethoxy-2-phenylacetophenone (Sigma-Aaldrich) were admixed at $100: 1$ weight ratio. BST nanoparticles (NanOxide HBS-2000, TPL Inc.) were dispersed in the liquid monomer solution at $2 \mathrm{wt} \%$ concentration. The resulting dispersion was coated with Meyer rod onto the AgNW/SWNT bilayer, followed by exposure to ultraviolet radiation to convert the liquid monomer into a solid crosslinked polymer matrix. The resulting SWNT/AgNW-nanocomposite film was peeled off the glass substrate.

PLEDs were fabricated on the nanocomposite electrode with a sheet resistance of $\sim 50 \mathrm{ohm} / \mathrm{sq}$, or ITO/glass substrates with a sheet resistance of $\sim 15 \Omega / \mathrm{sq}$. The ITO electrode was pre-cleaned and treated with oxygen plasma to increase the work function and decrease the surface roughness. Poly(3,4-ethylenedioxythiophene):poly(styrenesulfonate) (PEDOT:PSS) (Clevios VP Al 4083 from H. C. Starck Inc.) was spin-coated on the ITO glass substrates at $3000 \mathrm{rpm}$ for $60 \mathrm{~s}$ and then baked at $120^{\circ} \mathrm{C}$ for $15 \mathrm{~min}$. The resulting coating was approximately $40 \mathrm{~nm}$ thick. A green emissive polymer blend layer was then spin-coated from a solution of poly(vinylcarbazole) (PVK) $\left(\mathrm{M}_{\mathrm{W}}=1.1 \times 10^{6} \mathrm{~g} / \mathrm{mol}\right.$, Sigma-Aldrich), 1,3-bis[(4-tert-butylphenyl)-1,3,4oxidiazolyl]phenylene (OXD-7, Lumtech), and tris(2-(4-tolyl)pyridinato-N,C2) (Ir(mppy) $)_{3}$, American Dye Source) co-dissolved in chlorobenzene. The white emis sive polymer was provided by Cambridge Display Technology. The emissive layer, $75 \mathrm{~nm}$ in thickness, was annealed at $80^{\circ} \mathrm{C}$ for $30 \mathrm{~min}$. Finally, a thin layer of CsF $(1.0 \mathrm{~nm})$ and a layer of aluminum $(100 \mathrm{~nm})$ were successively deposited in a vacuum thermal evaporator through a shadow mask at $10^{-6}$ Torr. The resulting PLEDs had a sandwiched configuration of: glass/ITO/PEDOT:PSS (40 nm)/green or white emissive layer $(75 \mathrm{~nm}) / \mathrm{CsF}(1 \mathrm{~nm}) / \mathrm{Al}(100 \mathrm{~nm})$. The active area of the devices was $0.12 \mathrm{~cm}^{2}$. The device fabrication and testing were carried out in a nitrogen-filled dry box with oxygen and moisture levels both below $0.5 \mathrm{ppm}$. Luminance-voltage $(L-V)$ and current density-voltage $(J-V)$ characteristics were measured with a Keithley 2400 Semiconductor Characterization System and a calibrated silicon photodetector. The luminance intensity and electroluminescence (EL) spectra were further calibrated with a photoresearch PR-655.

1. Gather, M. C., Kohnen, A. \& Meerholz, K. White organic light-emitting diodes. Adv. Mater. 23, 233-248 (2011).

2. Xiao, L. et al. Recent progresses on materials for electrophosphorescent organic light-emitting devices. Adv. Mater. 23, 926-952 (2011).

3. Reineke, S. et al. White organic light-emitting diodes with fluorescent tube efficiency. Nature 459, 234-238 (2009).

4. Friend, R. H. et al. Electroluminescence in conjugated polymers. Nature 397, 121-128 (1999).

5. Wu, H. B. et al. Efficient single active layer electrophosphorescent white polymer light-emitting diodes. Adv. Mater. 20, 696-702 (2008).

6. Zou, J. et al. Simultaneous optimization of charge-carrier balance and luminous efficacy in highly efficient white polymer light-emitting devices. Adv. Mater. 23, 2976-2980 (2011).

7. Yu, Z. et al. Highly flexible silver nanowire electrodes for shape-memory polymer light-emitting diodes. Adv. Mater. 23, 664-668 (2011).

8. Yu, Z., Niu, X., Liu, Z. \& Pei, Q. Intrinsically stretchable polymer light-emitting devices using carbon nanotube-polymer composite electrodes. Adv. Mater. 23, 3989-3994 (2011).

9. Williams, E. L., Haavisto, K., Li, J. \& Jabbour, G. E. Excimer-based white phosphorescent organic light-emitting diodes with nearly $100 \%$ internal quantum efficiency. Adv. Mater. 19, 197-202 (2007).

10. Xiao, L., Su, S., Agata, Y., Lan, H. \& Kido, J. Nearly $100 \%$ internal quantum efficiency in an organic blue-light electrophosphorescent device using a weal electron transporting material with a wide energy gap. Adv. Mater. 21, 1271-1274 (2009).

11. Helander, M. G. et al. Chlorinated indium tin oxide electrodes with high work function for organic device compatibility. Science 332, 944-947 (2011).

12. Adachi, C., Baldo, M. A., Thompson, M. E. \& Forrest, S. R. Nearly $100 \%$ internal phosphorescence efficiency in an organic light-emitting device. J. Appl. Phys. 90, 5048-5051 (2001).

13. Chutinan, A., Ishihara, K., Asano, T., Fujita, M. \& Noda, S. Theoretical analysis on light-extraction efficiency of organic light-emitting diodes using FDTD and mode-expansion methods. Org. Electron. 6, 3-9 (2005).

14. Bulovic, V.et al. Weak microcavity effects in organic light-emitting devices. Phys. Rev. B 58, 3730-3740 (1998). 
15. Yu, G., Burrows, P. E., Venkatesh, S., Forrest, S. R. \& Thompson, M. E. Highexternal-quantum-efficiency organic light- emitting devices. Opt. Lett. 22, 396-398 (1997).

16. Ziebarth, J. M., Saafir, A. K., Fan, S. \& McGehee, M. D. Extracting light from polymer light-emitting diodes using stamped Bragg gratings. Adv. Funct. Mater. 14, 451-456 (2004).

17. Sun, Y. \& Forrest, S. R. Enhanced light out-coupling of organic light-emitting devices using embedded low-index grids. Nature Photon. 2, 483-487 (2008).

18. Choulis, S. A., Mathai, M. K. \& Choong, V. E. Influence of metallic nanoparticles on the performance of organic electrophosphorescence devices. Appl. Phys. Lett. 88, 213503 (2006)

19. Shiga, T., Fujikawa, H. \& Taga, Y. Design of multiwavelength resonant cavities for white organic light-emitting diodes. J. Appl. Phys. 93, 19-22 (2003).

20. Cho, T., Lin, C. \& Wu, C. Microcavity two-unit tandem organic light-emitting devices having a high efficiency. Appl. Phys. Lett. 88, 111106 (2006).

21. Ozbay, E. Plasmonics: merging photonics and electronics at nanoscale dimensions. Science 311, 189-193 (2006).

22. Atwater, H. A. \& Polman, A. Plasmonics for improved photovoltaic devices. Nature Mater. 9, 205-213 (2010).

23. Do, Y. R., Kim, Y., Song, Y. \& Lee, Y. Enhanced light extraction efficiency from organic light emitting diodes by insertion of a two-dimensional photonic crystal structure. J. Appl. Phys. 96, 7629-7636 (2004).

24. Agarwal, M., Sun, Y., Forrest, S. R. \& Peumans, P. Enhanced outcoupling from organic light-emitting diodes using aperiodic dielectric mirrors. Appl. Phys. Lett. 90, 241112 (2007).

25. Koo, W. et al. Light extraction from organic light-emitting diodes enhanced by spontaneously formed buckles. Nature Photon. 4, 222-226 (2010).

26. Wang, Z. B. et al. Unlocking the full potential of organic light-emitting diodes on flexible plastic. Nature Photon. 5, 753-757 (2011).

27. Koo, W. H. et al. Light extraction of organic light emitting diodes by defective hexagonal-close-packed array. Adv. Funct. Mater. 22, 3454-3459 (2012).

28. Madigan, C. F., Lu, M. H. \& Sturm, J. C. Improvement of output coupling efficiency of organic light-emitting diodes by backside substrate modification. Appl. Phys. Lett. 76, 1650-1652 (2000).

29. D’Andrade, B. W. \& Brown, J. J. Organic light-emitting device luminaire for illumination applications. Appl. Phys. Lett. 88, 192908 (2006).

30. Cheng, Y. H., Wu, J. L. \& Cheng, C. H. Enhanced light outcoupling in a thin film by texturing meshed surfaces. Appl. Phys. Lett. 90, 091102 (2007).

31. Peng, H. J., Ho, Y. L., Yu, X. J. \& Kwok, H. S. Enhanced coupling of light from organic light emitting diodes using nanoporous films. J. Appl. Phys. 96, 1649-1654 (2004).

32. Tsutsui, T., Yahiro, M., Yokogawa, H., Kawano, K. \& Yokogawa, M. Doubling coupling-out efficiency in organic light-emitting devices using a thin silica aerogel layer. Adv. Mater. 13, 1149-1152 (2001).

33. Shiang, J. J., Faircloth, T. J. \& Duggal, A. R. Experimental demonstration of increased organic light emitting device output via volumetric light scattering. J. Appl. Phys. 95, 2889-2895 (2004)

34. Li, F., Li, X., Zhang, J. \& Yang, B. Enhanced light extraction from organic lightemitting devices by using microcontact printed silica colloidal crystals. Org. Electron. 8, 635-639 (2007).

35. Moller, S. \& Forrest, S. R. Improved light out-coupling in organic light emitting diodes employing ordered microlens arrays. J. Appl. Phys. 91, 3324-3327 (2002)

36. Wei, M. \& Su, I. Method to evaluate the enhancement of luminance efficiency in planar OLED light emitting devices for microlens array. Opt. Exp. 12, 5777-5782 (2004)

37. Hsu, S., Lee, M., Lee, K. \& Wei, P. Extraction enhancement in organic light emitting devices by using metallic nanowire arrays. Appl. Phys. Lett. 92, 013303 (2008).

38. Li, L. et al. Efficient white polymer light-emitting diodes employing a silver nanowire-polymer composite electrode. Phys. Chem. Chem. Phys. 14, 14249-14254 (2012)
39. Gaynor, W. et al. Color in the corner: ITO-free white OLEDs with angular color stability. Adv. Mater. 25, 4006-4013 (2013).

40. Shiang, J. J. \& Duggal, A. R. Application of radiative transport theory to light extraction from organic light emitting diodes. J. Appl. Phys. 95, 2880-2888 (2004)

41. Van de Hulst, H. C. Multiple Light Scattering (Academic, New York, 1980).

42. Fang, C. et al. Nanoparticle stacks with graded refractive indices enhance the omnidirectional light harvesting of solar cells and the light extraction of lightemitting diodes. Adv. Funct. Mater. 23, 1412-1421 (2012).

43. Li, L. et al. Efficient flexible phosphorescent polymer light-emitting diodes based on silver nanowire-polymer composite electrode. Adv. Mater. 23, 5563-5567 (2011).

44. Liang, J., Li, L., Niu, X., Yu, Z. \& Pei, Q. Fully solution-based fabrication of flexible light-emitting device at ambient conditions. J. Phys. Chem. C 117, 16632-16639 (2013).

45. Kim, K. K. et al. Enhanced light extraction efficiency of GaN-based light-emitting diodes with $\mathrm{ZnO}$ nanorod arrays grown using aqueous solution. Appl. Phys. Lett. 94, 071118 (2009).

46. Chang, H. et al. Nano-particle based scattering layers for optical efficiency enhancement of organic light-emitting diodes and organic solar cells. J. Appl. Phys. 113, 204502 (2013).

47. Parker, I. D. Carrier tunneling and device characteristics in polymer lightemitting-diodes. J. Appl. Phys. 75, 1656-1666 (1994).

48. Jung, S. et al. Extremely flexible transparent conducting electrodes for organic devices. Adv. Energy Mater. 4, 1300474 (2014).

49. Wang, C., Hu, Y., Lieber, C. M. \& Sun, S. Ultrathin Au Nanowires and Their Transport Properties. J. Am. Chem. Soc. 130, 8902-8903 (2008).

50. Sun, Y. G., Gates, B., Mayers, B. \& Xia, Y. N. Crystalline silver nanowires by soft solution processing. Nano Lett. 2, 165-168 (2002).

51. Wu, H. et al. Electrospun metal nanofiber webs as high-performance transparent electrode. Nano Lett. 10, 4242-4248 (2010).

\section{Acknowledgments}

The work reported here was supported by the National Science Foundation (ECCS-1028412) and the Air Force Office of Scientific Research (Grant \# FA9550-12-1-0074). We thank Dr. Richard Wilson of Cambridge Display Technology (CDT) for providing the white emissive polymer

\section{Author contributions}

Q.P., L.L. and J.L. designed the nanocomposites. L.L. and J.L. carried out experiments and analyzed the data. L.L. did the FDTD simulation. Z.Y., S.C. and X.Z. optimized the PLED fabrication. X.N. assisted device testing and took the photographs. L.L. J.L. and Q.P. wrote the manuscript.

\section{Additional information}

Supplementary information accompanies this paper at http://www.nature.com/ scientificreports

Competing financial interests: The authors declare no competing financial interests. How to cite this article: Li, L. et al. A Solution Processed Flexible Nanocomposite Electrode with Efficient Light Extraction for Organic Light Emitting Diodes. Sci. Rep. 4, 4307; DOI:10.1038/srep04307 (2014).

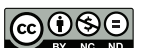

This work is licensed under a Creative Commons AttributionNonCommercial-NoDerivs 3.0 Unported license. To view a copy of this license, visit http://creativecommons.org/licenses/by-nc-nd/3.0 